\title{
Psoriasis as a disease associated with the immune system disorders
}

\author{
DOROTA CHOMICZEWSKA-SKÓRA ${ }^{1}$, EWA TRZNADEL-GRODZKA ${ }^{2}$, HELENA ROTSZTEJN ${ }^{3}$
}

${ }^{1}$ Nofer Institute of Occupational Medicine, Centre of Occupational Allergy and Environmental Health, Lodz, Poland

${ }^{2}$ Department of Dermatology and Pediatric Dermatology, Medical University of Lodz, Poland

${ }^{3}$ Department of Cosmetology, Medical University of Lodz, Poland

\begin{abstract}
The etiopathogenesis of psoriasis has not been fully elucidated. Nowadays, psoriasis is perceived as a multifactorial disease of genetic origin, in which complex immunological disorders concerning specific and non-specific defense mechanisms play a key role. Researchers emphasize the systemic and inflammatory character of the disease, its autoimmunological aspect and, above all, its dependence on T cells - Th1, Th17 and recently Th22. T cells with type 1 cytokine profile (Th1 and Tc1), Th17 and Th22 lymphocytes, regulatory $T$ cells, $N K$ and NK-T cells, antigen-presenting cells (dendritic cells, including Langerhans cells), keratinocytes, neutrophils, monocytes and makrophages are among the cells that take part in the pathogenesis of psoriasis.
\end{abstract}

Key words: psoriasis, lymphocytes, dendritic cells, cytokines.

(Centr Eur J Immunol 2013; 38 (1): 129-133)

Psoriasis is a chronic inflammatory skin disease in which epidermal hyperproliferation leads to the development of lesions of characteristic morphology. The process of epidermal hyperproliferation is associated with an increased number of cell divisions in the basal layer and abnormal keratinocyte maturation cycle [1]. The clinical manifestations of the disease are different and may include single papules and plaques, larger patches or generalized changes (psoriatic erythroderma) [2]. Psoriasis may also manifest as localized or generalized pustular lesions [1]. 5-40\% of psoriasis patients develop psoriatic arthritis [3]. Moreover, people with psoriasis are more often affected by such disorders as abdominal obesity, dyslipidemia, hypertension and diabetes (with insulin resistance). They are the components of metabolic syndrome which is connected with a greater risk of cardiovascular diseases [4-6].

The etiopathogenesis of psoriasis has not been fully elucidated yet. Experimental investigations and clinical observations indicate its high complexity, where genetic, immunological and environmental factors are involved [7]. Nowadays, psoriasis is perceived as a multifactorial disease of genetic origin, in which complex immunological disorders concerning specific and non-specific defense mechanisms play a key role $[8,9]$.

Researchers emphasize the systemic and inflammatory character of the disease, its autoimmunological aspect and, above all, its dependence on T cells - Th1, Th17 and recently Th22 $[10,11]$. It is thought that immunological disorders in people who are genetically predisposed lead to initiating and sustaining of skin inflammation, resulting in abnormal differentiation and excessive proliferation of keratinocytes [12]. Cellular and humoral responses of the immune system are equally important in this process. T cells with type 1 cytokine profile (Th1 and Tc1), Th17 and Th22 lymphocytes, regulatory T (Treg) cells, NK and NK-T cells, antigenpresenting cells (dendritic cells, including Langerhans cells), keratinocytes, neutrophils, monocytes and macrophages are among the cells that take part in the pathogenesis of psoriasis $[13,14]$.

Other important factors include:

- interleukines: IL-1, IL-2, IL-6, IL-8, IL-12, IL-17, IL-20, IL-22, IL-23, tumor necrosis factor $\alpha(\mathrm{TNF}-\alpha)$ and interferon $\gamma(\mathrm{IFN}-\gamma)$,

- some growth factors: vascular endothelial growth factor (VEGF), keratinocyte growth factor (KGF) [9, 11, 15],

Correspondence: Prof. Helena Rotsztejn, Department of Cosmetology, Medical University of Lodz, 1 Muszynskiego Street, 90-151 Lodz, Poland, tel. +48 604180 044, fax +48 4267791 14, e-mail: helena.rotsztejn @umed.lodz.pl 
- chemokines participating in cell migration: CCL17 (TARC), CXCL9 (MIG), CXCL10 (IP10), CCL22 (MDC), CCL5 (RANTES), CXCR2, CCR4, CCL27, CCR10, CCL20 (MIP3 $\alpha$ ), CCL19 (MIP3 $\beta$ ) and CCR6 [2],

- adhesions molecules supporting leukocytes migration: intercellular adhesion molecule-1 (ICAM-1), vascular cell adhesion molecule 1 (VCAM-1), E-selectins and vascular adhesion protein 1 (VAP-1) [11].

Individual factors participate in different stages of psoriasis development [15]. T cells, keratinocytes and their interaction by means of cytokines are particularly important. It is emphasized that both innate and adaptive immunological mechanisms may in turns maintain inflammation in the epidermis and consequently the response of keratinocytes [16]. However, a uniform pathogenetic model of the disease, covering all potential factors, has not been provided [9].

An important role of the immune system in the formation of psoriatic lesions is confirmed by numerous experimental models with the use of transplantation of human skin in animals with immunological defects [17]. Pharmacotherapies using the suppressive or modifying activity of such drugs as cyclosporine or biological drugs have also proved to be effective in the treatment of psoriasis $[17,18]$. Moreover, the immunological base of the disease may be confirmed by the development or regression of psoriatic lesions in recipients after the transplantation of bone marrow which is a source of precursors for various cells of the immune system [19].

\section{The importance of non-specific immune mechanisms}

Stimulation of non-specific response mechanisms in psoriasis may be due to inadequate (excessive) reaction of the immune system to unidentified environmental triggers, such as trauma or infectious agents penetrating the epidermal barrier [7, 16, 20]. The examinations of psoriatic lesions have shown the presence of cellular and humoral effectors of non-specific response, including a number of factors released by the stimulation of keratinocytes and immune cells [21]. Research has shown an increase in the number and activation of neutrophils, monocytes and mocrophages, dendritic cells, NK and NK-T cells, and hyperplasia of keratinocytes $[6,16,20]$. Increased expression of humoral factors has also been observed:

- receptors involved in microbial recognition - Toll-like receptors (TLR - TLR1, TLR2) [22],

- heat shock proteins (HSP) [23],

- komplement components [24],

- cytokines (TNF- $\alpha$, IFN- $\gamma$, IL-1, IL-6, IL-12, IL-15, IL-18),

- chemokines IL-8,

- antimicrobial peptides and proteins (AMPs) [16].

The peptides which are potentially important in psoriasis include $\beta$-defensins (hBD-2, hBD-3) [7], cathelicidins (IL-37), S100 proteins and lysozyme [7, 17].
Microorganisms, by means of so-called pattern recognition receptors (PRR), such as TLRs or type C lectins, may play an important role in the initiation of the immune response [7, 22, 25]. As a result of stimulation of these receptors, keratinocytes and immune cells secrete cytokines, chemokines, protease inhibitors, neuropeptides and peptides, including endogenous antimicrobial proteins and peptides [6]. Originally triggered innate factors further contribute to the activation of immune cells, their recruitment to the skin and are associated with the engagement of specific immune mechanisms with the participation of T cells $[7,16]$.

In psoriasis, keratinocytes perform immune function and they are a source of proinflammatory cytokines, including TNF- $\alpha$, IL- 1 and IL- 6 as well as chemokines, including IL-8 which induces chemotaxis of neutrophils and lymphocytes into the epidermis [16]. It has been shown that epidermal cells in psoriatic lesions, due to cytokine release, have the ability to activate $\mathrm{CD} 4+\mathrm{T}$ cells, whereas normal keratinocytes do not produce such an effect [26].

Neutrophils accumulate in psoriatic epidermis and perivascular space, creating clusters observed microscopically, called Munro's microabscesses [16]. These cells are present in psoriatic lesions rather in the early stages of their development [17]. It is believed that they, by means of secreted elastases, may also contribute to epidermal hyperproliferation [27]. An increased number of NK and NK-T cells in the lesions and serum of patients suggests their participation in psoriasis process [28]. The same phenomenon applies to macrophages in psoriatic skin [29].

Dendritic cells may be involved in the non-specific response mechanisms in psoriasis by recognizing pathogens through TLRs and CD1 and secretion of proinflammatory cytokines and chemokines [30]. Increased numbers of dendritic cells have been reported in literature. In addition, many of these cells correspond phenotypically to mature cells, having such receptors as CD80, CD83, CD86 and able to stimulate lymphocytes, thus providing a link between specific and non-specific immune mechanisms [31]. It has been shown that also in the clinically intact skin in patients with psoriasis, dendritic cells exhibit stimulating activity [32].

\section{The importance of specific immune mechanisms}

$\mathrm{T}$ cells are the elements of specific immune response and play a key role in psoriasis. Significantly more different populations of $\mathrm{T}$ cells are found in the lesions than in healthy skin. These are CD4+ CD45RO+, CD4+ CD25+, CD8+ CD45RO+, CD8+ CD25+ lymphocytes [33]. In the dermis, CD4+ T cells ( $\mathrm{Th}-$ helper $\mathrm{T}$ cells) are more numerous than CD8+ T cells ( $\mathrm{Tc}-$ cytotoxic $\mathrm{T}$ cells), while the latter predominate in the epidermis [34]. In the psoriatic process, the circulating $\mathrm{T}$ cells are stimulated and next recruited to the skin, where they participate in the formation of skin lesions [35]. 
In psoriatic skin there are more CD45RO+ memory $\mathrm{T}$ cells formed as a result of antigen stimulation than CD45RA+ naive T cells [34]. Their activation requires prior interaction with antigen-presenting cells (APC), which include Langerhans cells and other dendritic cells [17].

Sabat et al. proposed a model consisting of three phases of development of the immune response in psoriasis sensitization, silence and effector phase. In their course, initially asymptomatic clinical events precede immune cells migration to the skin and the response from keratinocytes [1].

In the first phase, skin dendritic cells stimulated by various not fully identified antigens and autoantigens, undergo transformation and maturation and present antigens to $\mathrm{T}$ cells in the regional lymph nodes.

It is believed that bacterial superantigens [37], streptococcal M protein [38], retroviral antigens, human papillomavirus (HPV) [39], neuropeptides, such as substance $\mathrm{P}$ [40], keratin-derived peptides [9] and stratum corneum autoantigens and keratin 17 [16] may have a potential impact on the development of psoriasis.

As a result of the interaction with dendritic cells, naive $\mathrm{T}$ cells differentiate into effector or memory cells. CD4+ T cells produce populations of Th1 or Th17 and CD8+ T cells produce Tc1 - crucial in psoriasis helper cells and cytotoxic cells of type 1 cytokine profile (secreting IL-2, TNF- $\alpha$ and IFN- $\gamma$ ) and helper cells of type 17 cytokine profile (releasing IL-6, IL-17 and IL-22) [15, 41]. Then lymphocytes migrate from the lymph nodes via lymphatic and blood vessels and settle into the skin. This process is possible thanks to the receptors and adhesive particles present on the surface of the cells and endothelium.

Cutaneous lymphocyte-associated antigen (CLA), called skin-homing receptor plays a special role on the part of lymphocytes. Chemokines (MIP-5 $\alpha$, RANTES, macrophagederived chemokine, monokine induced by IFN- $\gamma$, inducible protein-10) and chemokine receptors (CCR6, CCR5, CCR4, CXCR5) are also involved in the migration of T cells in psoriasis [40].

After the initial lymphocyte stimulation, clinically asymptomatic phase begins and is characterized by the presence of memory cells in the blood and tissues [15].

In the last, effector and symptomatic chase, as a result of antigenic restimulation, the previously sensitized $\mathrm{T}$ cells migrate to the skin and are stimulated. This phenomenon is accompanied by infiltration of other immune cells, such as monocytes and macrophages, dendritic cells and neutrophils. Lymphocyte and other cell interactions and secreted inflammatory mediators cause hyperproliferation and abnormal differentiation of keratinocytes and formation of clinically observed psoriatic lesions $[15,40]$.

Some authors point to the involvement of autoimmune mechanisms in the pathogenesis of psoriasis. As mentioned above, the stratum corneum autoantigens may play a role in initiating the immune processes [16].
According to Nickoloff and Nestle, during immune synapse formation and autoantigen presentation to $\mathrm{T}$ cells by dendritic cells, a mutation within the receptors responsible for the transmission of signals between the cells does not allow for the elimination of autoreactive T cells. Lymphocytes may then interact with antigen-presenting cells and keratinocytes and induce plaque formation [2]. As mentioned above, the pathogenetic process in psoriasis may involve different populations of lymphocytes, including CD4+CD25+ regulatory T cells. Sugiyama et al. point to their abnormal suppressive activity in this disease [42].

Summing up, different proinflammatory stimuli lead to the interaction of dendritic cells and T cells, resulting in immune synapse formation between the cells and subsequent activation of antigen-specific T cells. This results in the release of cytokines, chemokines and growth factors, which initiate the proliferation of epidermal cells and continue to stimulate the activation of T cells and antigen-presenting cells [10]. Th1 and Th17 lymphocytes, in particular, but also macrophages and neutrophils are responsible for the release of proinflammatory mediators, the maintenance of inflammation in the skin and development of psoriatic lesions $[10,43]$.

\section{The importance of cytokines}

Proinflammatory cytokines are involved in every phase of the development of psoriasis. They contribute to the establishment of pathogenic vicious circle, in which keratinocytes, endothelium and immune cells release inflammatory mediators, stimulate each other, maintaining the process of chronic inflammation [2]. Type 1 cytokines (TNF- $\alpha$, IFN- $\gamma$, IL-2), type 17 cytokines (IL-6, IL-17 and IL-22) and IL-23 are the most important in psoriasis [44].

Recently, the authors have also pointed to the role of IL-20, IL-15 and IL-26 [11]. Elevated levels of many cytokines are found in psoriatic lesions (TNF- $\alpha$, IL-6, IL-17, IL-20, IL-22) [44-46], in the serum (TNF- $\alpha$, IFN- $\gamma$, IL-6, IL-8, IL-12, IL-17, IL-18, IL-20, IL-22) [4, 47] and in the synovium in psoriatic arthritis (TNF- $\alpha$ ) [45]. The role of cytokines in the pathogenesis of psoriasis is confirmed by the effectiveness of therapies directed against different humoral factors, such as TNF- $\alpha$ [48], IL-12 and IL-23 [49].

Tumor necrosis factor $\alpha$ is secreted by keratinocytes, dendritic cells, T cells, NK cells, neutrophils, monocytes and macrophages [50]. For many years this factor was regarded as a central cytokine in psoriasis. Effects of its activity include stimulation of the synthesis and release of proinflammatory cytokines [40], stimulation of the proliferation and differentiation of many cells [51], stimulation of dendritic cell maturation [52], increasing the expression of adhesive molecules on endothelial cells and keratinocytes, decreasing the expression of E-cadherins, which 
facilitates the migration of immune cells, and stimulation of epidermal cell division [40].

Interferon $\gamma$ is released by T cells, NK cells, macrophages and keratinocytes [2]. It is an immunoregulatory cytokine which increases the activity of antigen-presenting cells, increases the expression of adhesive molecules on endothelial cells and keratinocytes, stimulates the release of certain cytokines and contributes to increase in the number of receptors for TNF- $\alpha$ on cells. Interferon $\gamma$, as an inhibitor of the proliferation of keratinocytes, may influence this process in psoriatic epidermis [40].

Interleukin 6 is produced by, inter alia, keratinocytes, endothelial cells and white blood cells migrating to the skin. It contributes to the psoriatic epidermal hyperplasia and may affect the function of immune cells. For example, it may stimulate the differentiation of Th17 cells, which, by means of IL-17, stimulate the release of IL-6 from keratinocytes [46].

Interleukin 17 , synthesized by Th cells, plays a key role in inducing and maintaining inflammation in psoriasis, stimulates the synthesis of proinflammatory cytokines, such as IL-1, IL-6 and GM-CSF, inter alia, through endothelial cells, monocytes and macrophages. It stimulates the secretion of IL-8 and other chemokines by kerationcytes [44, 51]. It also influences neutrophils by activating them [49].

Interleukin 22, secreted mainly by Th17 cells and additionally by other populations of lymphocytes, is responsible for epidermal hyperplasia and hypogranulosis and inhibits terminal differentiation of keratinocytes and also regulates their migration process. Moreover, it stimulates the release of inflammatory mediators - cytokines, chemokines, acute phase proteins and antimicrobial peptides [25, 44].

Interleukin 20 is released by monocytes, dendritic cells and keratinocytes. Its effect on keratinocytes is similar to that of IL-22 [4].

Interleukin 23, produced by dendritic cells and macrophages, is responsible for the stimulation of $\mathrm{T}$ cells [44]. Probably, it also is responsible for the formation of inflammatory infiltrates of various epidermal immune cells, promotes epidermal acanthosis [53] and causes the release of TNF- $\alpha$ [44]. In recent years, an important role in the pathogenesis of psoriasis has been attributed to the interrelation between Th17 cells and IL-23 [49]. Interleukin 23 stimulates Th17 cells to produce IL-17 and IL-22, which in turn induce activation and proliferation of keratinocytes. Activated epidermal cells become a source of cytokines, chemokines and antimicrobial peptides, resulting in an influx of immune cells into the epidermis and their subsequent activation [49].

This work was supported by Medical University of Lodz grant statute no 503/3-066-02/503-01 (to E. Budzisz).

\section{References}

1. Kemény L, Ruzicka T, Braun-Falco O (1990): Dithranol: a review of the mechanism of action in the treatment of psoriasis vulgaris. Skin Pharmacol 3: 1-20.

2. Nickoloff BJ, Nestle FO (2004): Recent insights into the immunopathogenesis of psoriasis provide new therapeutic opportunities. J Clin Invest 113: 1664-1675.

3. Christophers E, Barker JN, Griffiths CE, et al. (2010): The risk of psoriatic arthritis remains constant following initial diagnosis of psoriasis among patients seen in European dermatology clinics. J Eur Acad Dermatol Venereol 24: 548-554.

4. Gottlieb AB, Chao C, Dann F (2008): Psoriasis comorbidities. J Dermatolog Treat 19: 5-21.

5. Gisondi P, Tessari G, Conti A, et al. (2007): Prevalence of metabolic syndrome in patients with psoriasis: a hospital-based case-control study. Br J Dermatol 157: 68-73.

6. Szponar-Bojda A, Krasowska D, Pietrzak A, Chodorowska G (2012): Metabolic syndrome in psoriasis. Postep Derm Alergol 29: 356-362.

7. Büchau AS, Gallo RL (2007): Innate immunity and antimicrobial defense systems in psoriasis. Clin Dermatol 25: 616-624.

8. Elder JT (2009): Genome-wide association scan yields new insights into the immunopathogenesis of psoriasis. Genes Immun 10: 201-209.

9. Das RP, Jain AK, Ramesh V (2009): Current concepts in the pathogenesis of psoriasis. Indian J Dermatol 54: 7-12.

10. Prinz JC (2010): From bench to bedside - translational research in psoriasis. J Eur Acad Dermatol Venereol 24 (Suppl 6): 1-4.

11. Michalak-Stoma A, Pietrzak A, Szepietowski JC, et al. (2011): Cytokine network in psoriasis revisited. Eur Cytokine Netw 22: 160-168.

12. Nickoloff BJ, Schröder JM, von den Driesch P, et al. (2000): Is psoriasis a T-cell disease? Exp Dermatol 9: 359-375.

13. Rotsztejn H, Zalewska A, Trznadel-Budźko E, et al. (2005): Influence of systemic photochemotherapy on regulatory $\mathrm{T}$ cells and selected cytokine production in psoriatic patients - a pilot study. Med Sci Monit 11: CR594-8.

14. Chomiczewska D, Trznadel-Grodzka E, Kaczorowska A, Rotsztejn H (2009): Znaczenie komórek Langerhansa w układzie immunologicznym skóry. Pol Merk Lek XXVI, 153: 173-177.

15. Sabat R, Philipp S, Höflich C, et al. (2007): Immunopathogenesis of psoriasis. Exp Dermatol 16: 779-798.

16. Bos JD, de Rie MA, Teunissen MB, Piskin G (2005): Psoriasis: dysregulation of innate immunity. Br J Dermatol 152: 1098-1107.

17. van de Kerkhof PC (2007): The evolution of the psoriatic lesion. Br J Dermatol 157: 4-15.

18. Adamski Z, Dudziak M, Zakrzewska K (2011): Etanercept in dermatological practice - authors' own experience in the treatment of psoriasis vulgaris and psoriatic arthritis. Postep Derm Alergol 28: 435-441.

19. Eedy DJ, Burrows D, Bridges JM, Jones FG (1990): Clearance of severe psoriasis after allogenic bone marrow transplantation. Br Med J 300: 908.

20. Sanchez AP (2010): Immunopathogenesis of psoriasis. An Bras Dermatol 85: 747-749.

21. Sweeney CM, Tobin AM, Kirby B (2011): Innate immunity in the pathogenesis of psoriasis. Arch Dermatol Res 303: 691-705.

22. Baker BS, Ovigne JM, Powles AV, et al. (2003): Normal keratinocytes express Toll-like receptors (TLRs) 1, 2 and 5: 
modulation of TLR expression in chronic plaque psoriasis. Br J Dermatol 148: 670-679.

23. Curry JL, Qin JZ, Bonish B, et al. (2003): Innate immune-related receptors in normal and psoriatic skin. Arch Pathol Lab Med 127: 178-186.

24. Pasch M, Bos JD, Asghar SS (1998): Activation of complement in psoriasis. Clin Exp Dermatol 23: 189-192.

25. Gliński W (2009): Antimicrobial peptides in the pathogenesis of psoriasis and atopic dermatitis. Przegl Dermatol 96: 115-120 [article in Polish].

26. Chang EY, Hammerberg C, Fisher G, et al. (1992): T-cell activation is potentiated by cytokines released by lesional psoriatic, but not normal epidermis. Arch Dermatol 128: 1479-1485.

27. Rogalski C, Meyer-Hoffert U, Proksch E, Wiedow O (2002): Human leukocyte elastase induces keratinocyte proliferation in vitro and in vivo. J Invest Dermatol 118: 49-54.

28. Cameron AL, Kirby B, Fei W, Griffiths CE (2002): Natural killer and natural killer-T cells in psoriasis. Arch Dermatol Res 294: 363-369.

29. Cabrijan L, Lipozencić J, Batinac T, et al. (2009): The role of CD4 and CD8 lymphocytes and macrophages in psoriasis vulgaris. Acta Dermatovenerol Croat 17: 162-165.

30. Nedoszytko B, Roszkiewicz J (2007): The role of the subpopulations of dendritic cells in psoriasis. Post Dermatol Alergol 6: 263-270 [article in Polish].

31. Abrams JR, Kelley SL, Hayes E, et al. (2000): Blockade of T lymphocyte costimulation with cytotoxic T lymphocyte-associated antigen 4-immunogobulin (CTLA4 Ig) reverses the cellular pathology of psoriatic plaques, including the activation of keratinocytes, dendritic cells and endothelial cells. J Exp Med 192: 681-693.

32. Zhou X, Krueger JG, Kao MC, et al. (2003): Novel mechanism of T-cell and dendritic cell activation revealed by profiling of psoriasis on the 63,100-element oligonucleotide array. Physiol Genomics 13: 69-78.

33. Bovenschen HJ, van Vlijmen-Wilems IM, van de Kerkhof PC, van Erp PE (2006): Identification of lesional CD4+ CD25+ Foxp3+ regulatory $\mathrm{T}$ cells in psoriasis. Dermatology 213: 111-117.

34. Krueger JG (2002): The immunologic basis for the treatment of psoriasis with new biological agents. J Am Acad Dermatol 46: $1-23$.

35. Baker BS, Swain AD, Fry L, Valdimarsson H (1984): Epidermal $\mathrm{T}$ lymphocytes and HLA-DR expression in psoriasis. $\mathrm{Br}$ J Dermatol 110: 555-564.

36. Bos JD, Hagenaars C, Das PK, et al. (1989): Predominance of "memory" T cells (CD4+, CDw29+) over "naive" T cells $(\mathrm{CD} 4+, \mathrm{CD} 45 \mathrm{R}+)$ in both normal and diseased human skin. Arch Dermatol Res 281: 24-30.

37. Valdimarsson H, Baker BS, Jónsdóttir I, et al. (1995): Psoriasis: a T-cell mediated autoimmune disease induced by streptococcal superantigens? Immunol Today 16: 145-149.

38. Sigmundsdottir H, Sigurgeirsson B, Troye-Blomberg M, et al. (1997): Circulating T-cells of patients with active psoriasis respond to streptococcal M-peptides sharing sequences with human epidermal keratins. Scand J Immunol 45: 688-697.

39. Majewski S, Jablonska S (2003): Possible involvement of epidermodysplasia verruciformis human papillomaviruses in the immunopathogenesis of psoriasis: a proposed hypothesis. Exp Dermatol 12: 721-728.

40. Lee MR, Cooper AJ (2006): Immunopathogenesis of psoriasis. Australas J Dermatol 47: 151-159.

41. Austin LM, Ozawa M, Kikuchi T, et al. (1999): The majority of epidermal T-cells in psoriasis vulgaris lesions can produce type 1 cytokines, interferon-gamma, interleukin-2 and tumor necrosis factor-alpha, defining TC1 (cytotoxic T lymphocyte) and TH1 effector populations: a type 1 differentiation bias is also measured in circulating blood T cells in psoriatic patients. J Invest Dermatol 115: 752-759.

42. Sugiyama H, Gyulai R, Toichi E, et al. (2005): Dysfunctional blood and target tissue CD4+ CD25high regulatory $\mathrm{T}$ cells in psoriasis: mechanism underlying unrestrained pathogenic effector T cell proliferation. J Immunol 174: 164-173.

43. Lowes MA, Lew W, Krueger JG (2004): Current concepts in the immunopathogenesis of psoriasis. Dermatol Clin 22: 349-369.

44. Coimbra S, Oliveira H, Reis F, et al. (2010): Interleukin (IL)-22, IL-17, IL-23, IL-8, vascular endothelial growth factor and tumour necrosis factor-a levels in patients with psoriasis before, during and after psoralen - ultraviolet A and narrowband ultraviolet B therapy. Br J Dermatol 163: 1282-1290.

45. Ritchlin C, Haas-Smith SA, Hicks D, et al. (1998): Patterns of cytokine production in psoriatic synovium. J Rheumatol 25 : 1544-1552.

46. Fujishima S, Watanabe H, Kawaguchi M, et al. (2010): Involvement of IL-17F via the induction of IL-6 in psoriasis. Arch Dermatol Res 302: 499-505.

47. Arican O, Aral M, Sasmaz S, Ciragil P (2005): Serum levels of TNF- $\alpha$, IFN- $\gamma$, IL-6, IL-8, IL-12, IL-17 and IL-18 in patients with active psoriasis and correlation with disease severity. Mediators Inflamm 5: 273-279.

48. Menter A (2009): The status of biologic therapies in the treatment of moderate to severe psoriasis. Cutis 84 Suppl. 4: 14-24.

49. Di Cesare A, Di Meglio P, Nestle FO (2009): The IL-23/Th17 axis in the immunopathogenesis of psoriasis. J Invest Dermatol 129: 1339-1350.

50. Schottelius AJ, Moldawer LL, Dinarello CA, et al. (2004): Biology of tumor necrosis factor- $\alpha$ - implications for psoriasis. Exp Dermatol 13: 193-222.

51. Pietrzak AT, Zalewska A, Chodorowska G, et al. (2008): Cytokines and anticytokines in psoriasis. Clin Chim Acta 394: 7-21.

52. Gottlieb AB, Chamian F, Masud S, et al. (2005): TNF inhibition rapidly down-regulates multiple proinflammatory pathways in psoriasis plaques. J Immunol 175: 2721-2729.

53. Torti DC, Feldman SR (2007): Interleukin-12, interleukin23, and psoriasis: current prospects. J Am Acad Dermatol 57: 1059-1068. 